\title{
Determinación de capacidad de carreteras en Cuenca (Ecuador) y comparación con el manual de capacidad HCM
}

\author{
Fabián Carpio $^{1^{*}, \text { Juan Avilés }}{ }^{2}$, Diana Morillo ${ }^{3}$ \\ ${ }^{1}$ Facultad de Ingeniería Civil, Universidad de Cuenca, Cuenca, Ecuador. \\ ${ }^{2}$ Facultad de Ingeniería Civil y Facultad de Arquitectura, Universidad de Cuenca, Cuenca, Ecuador. \\ ${ }^{3}$ Departamento de Biociencias, Facultad de Química, Universidad de Cuenca, Cuenca, Ecuador. \\ *Autor de correspondencia: faramircarpio@ hotmail.com \\ Fecha de recepción: 13 de septiembre 2017 - Fecha de aceptación: 18 de junio 2018
}

\section{RESUMEN}

Se realizó un estudio transversal en las secciones de 6 vías de doble carril en la Ciudad de Cuenca (Ecuador), en el período agosto 2015 a febrero 2016. La capacidad de la carretera se calculó utilizando, por un lado, las observaciones de campo y, por otro lado, aplicando el Manual de Capacidad de Carreteras (HCM). El método de HCM sobreestimó la capacidad de la carretera, en promedio con un $58 \%$, en comparación con la capacidad de la carretera obtenida utilizando los datos de campo, los cuales fueron obtenidos a través del método conocido como "observador moviéndose". La sobreestimación de la capacidad de la carretera con el HCM se debe al hecho de que los parámetros en este método no representan realmente los parámetros locales de nuestras avenidas. La comparación entre ambos métodos revela que el método HCM, derivado de las condiciones del tráfico de autopistas de los Estados Unidos, no es aplicable a las condiciones de la carretera-tráfico en Cuenca.

Palabras clave: Capacidad vial, flujo vehicular, velocidad de flujo libre, nivel de servicio.

\begin{abstract}
A transverse study was carried out in cross sections of 6 double lane roads in the City of Cuenca (Ecuador), in the period August 2015 to February 2016. The road capacity was calculated using at one hand field observations and at the other hand applying the Highway Capacity Manual (HCM). The HCM approach overestimated the road capacity on average with 58\% as compared to the road capacity obtained using field data, field data was collected using the "moving observer" method. The overestimation of the road capacity using HCM occur since the parameters in this method not really represent local avenue parameters. Comparison between both methods clearly reveals that the HCM method, derived for American highway-traffic conditions, is not applicable to the road-traffic conditions in Cuenca.
\end{abstract}

Keywords: Road capacity, traffic flow, free flow speed, service level.

\section{INTRODUCCIÓN}

La planificación, dimensionamiento y evaluación de carreteras depende de la determinación de su capacidad. La capacidad de una carretera según la definición presentada en el Highway Capacity Manual, HCM por sus siglas en inglés, se describe como “...la máxima tasa horaria (volumen de automotores) a la cual los vehículos razonablemente pueden esperar atravesar una sección transversal de un carril en una vía durante un tiempo dado, bajo las condiciones de tráfico predominantes..." (Hoogendoorn \& Bovy, 2001). No obstante, por mucho tiempo los investigadores han reconocido que esta definición resulta inadecuada e impracticable para un recurso vial, ya que, dependiendo de las condiciones de tráfico, empiezan a influir aspectos subjetivos como lo es la idiosincrasia del conductor 
(Elefteriadou \& Lertworawanich, 2003; Lertworawanich \& Elefteriadou, 2004; Kondyli, George, Elefteriadou, \& Bonyani, 2017).

En la mayoría de los países del mundo se usa la metodología planteada en el HCM (Maldonado, Herz, \& Galarraga, 2012), en cuyas aplicaciones, orientadas a la adaptación de los procedimientos en las condiciones locales, han llevado a plantear metodologías alternativas como es el caso de Sudáfrica, donde se proponen nuevas medidas de eficacia y aplicación de modelos macroscópicos (Van As \& Van Niekerk, 2004; Van As, 2007). En España, Romana \& Pérez (2006) presentaron una manera alternativa, planteando la definición de una velocidad umbral, basada en la expectativa de los usuarios. Estudios realizados en Brasil (Bessa Jr., 2009), utilizando una metodología similar a la de este trabajo, presentan modelos particulares para las relaciones fundamentales de flujo-velocidad y flujo-porcentaje de tiempo en seguimiento. En cuanto a la metodología HCM, a partir de la publicación de la versión HCM 2000 surgieron diferentes estudios cuestionando las ecuaciones y resultados, principalmente las diferencias observadas entre el análisis conjunto de ambos carriles y el análisis direccional (Luttinen, 2001). De igual manera, el mismo análisis de inaplicabilidad del manual HCM lo hacen los autores Arun, Velmurugan, \& Errampalli (2013). En su estudio, esos autores declararon que en la India las condiciones de las localidades debilitan la aplicación de los métodos HCM.

El modelo propuesto por el HCM se basa en la determinación de la Velocidad de Flujo Libre (VFL), proponiendo una ecuación para su determinación, haciendo uso de esta, y, con la ayuda de gráficas, se puede predecir la capacidad de la vía, es decir el punto de inflexión de la curva de la relación fundamental de tránsito (TRB, 2000a; Cal, Mayor, \& Cárdenas, 1996). HCM plantea VFL como una función de diversas variables que pueden cambiar según la localidad analizada, esta es quizá la razón del porque sus valores, obtenidos mediante el método HCM, son susceptibles de variaciones debido a la influencia de estas variables que no pueden ser cuantificadas. Entre estas se encuentran: las condiciones geométricas de la vía, que condicionan al conductor a decidir su velocidad de viaje, el factor humano o el comportamiento propio del conductor, e inclusive la cultura de la ciudad en la que se realiza el estudio. Además de los artículos mencionados, también se cuenta con estudios europeos (Moreno et al., 2016) y latinoamericanos (Maldonado et al., 2012), que también han identificado la necesidad de ajustar el modelo HCM a la realidad de la región en donde será aplicado, esto, mediante la definición de las variables influyentes para VFL. A lo dicho se suma el hecho de que la propia HCM hace referencia a la limitada aplicabilidad de sus ecuaciones para sitios donde las velocidades de circulación son bajas. Sin embargo, al carecer de una herramienta de evaluación para la capacidad vial en nuestro medio, nos vemos en la necesidad de partir siempre desde las sugerencias del HCM. La falta de información sobre las variables influyentes del VFL podría confluir en decisiones alejadas de la real necesidad de la estructura vial, generando sobre o infra dimensionamiento de esta infraestructura, produciendo probablemente inversiones inadecuadas.

En la ciudad de Cuenca, no se han descrito las características locales involucradas para la determinación de VFL y no se ha comprobado la exactitud de las gráficas propuestas por el método HCM. En el presente análisis comparativo se ha utilizado el método empleado por HCM para autopistas multi-carriles ya que en este método es donde se puede determinar la capacidad, mientras que los métodos para vías urbanas o de caminos direccionales asumen directamente una capacidad por carril de $1,700 \mathrm{veh} / \mathrm{hora}$, por lo que hemos elegido a las secciones de las avenidas urbanas de Cuenca con mayor similitud a las condiciones originales del método HCM. Tampoco se posee un método alternativo de evaluación de la capacidad vial. Ante la falta de esta información, el método HCM se aplicará para la determinación de la capacidad de las carreteras de la ciudad de Cuenca, luego, estos resultados serán contrastados con los datos reales obtenidos en campo. Esto permitirá analizar las diferencias.

\section{MATERIALES Y MÉTODOS}

El presente estudio se enfoca en el análisis comparativo de la capacidad vial de seis avenidas multicarril (doble carril en ambos sentidos) de la ciudad de Cuenca, mediante la determinación con datos reales, obtenidos en el campo, y comparados con los valores resultado de la aplicación del modelo propuesto por HCM. Los datos de campo obtenidos durante horas pico (11:00 y 13:00), incluyendo el tráfico de 
vehículos pesados, fueron recolectados en secciones de vía con características similares entre ellas, para de esta manera condicionar que los resultados dependan, en lo posible, únicamente de las condiciones de flujo vehicular, mas no de las condiciones físicas de las avenidas. Los datos nos permitieron relacionar la velocidad, densidad del tráfico y la tasa de flujo (Fig. 1), reflejando los efectos que producen en el conductor factores subjetivos, y por ende de dificultosa cuantificación, como son: la proximidad de otros vehículos, las condiciones de la calzada, el ancho de los carriles, ancho de parterre, tráfico pesado, entre otros, para luego compararlas con la capacidad obtenida a partir de la fórmula, tablas y gráficas que sugiere el método HCM.

Los resultados de la comparación de los valores de capacidad obtenidos por ambos métodos se expresaron mediante una razón mostrada en la ecuación (1), con la finalidad de establecer parámetros de ajuste en la aplicación final de los valores a nuestras avenidas.

$$
r=\frac{\text { capacidad obtenida en campo }}{\text { capacidad propuesta por HCM }}
$$

Para obtener los datos de campo, se realizó un estudio observacional en una sección transversal de una muestra de 6 tramos de carreteras de similares características en su capa de rodadura, ancho de carriles, ancho de parterre y demás características físicas concordantes con las explicadas por el método HCM para carreteras multi-carriles. Estos datos fueron recolectados durante el período agosto 2015 a febrero 2016, durante las horas pico de tráfico vehicular e incluyendo el tráfico pesado. El método que propone HCM se basa en gráficas desarrolladas en base a la relación fundamental del tránsito, donde el flujo de vehículos por hora es igual a la densidad de vehículos en un kilómetro por la velocidad del flujo (Cal et al., 1996). Las gráficas fueron creadas y categorizadas para tres tipos de vías: autopistas, carreteras multi-carriles y vías bidireccionales. Los tramos de carreteras analizados fueron aquellos que cumplieron con las características de carreteras multi-carriles.

En la ciudad de Cuenca, 126,000 vehículos fueron matriculados en el año 2016 (INEC, 2013), y la existencia de carreteras tipo multi-carril representan las principales arterias que conectan los puntos importantes de la ciudad. Por ello, los tramos de carreteras analizados fueron aquellos que cumplieron con las características en el modelo del HCM. Estas secciones viales guardan entre si una similitud muy estrecha, lo que hizo posible un análisis de segmento de recta de avenidas con igualdad de calzada y de condiciones geométricas muy homogéneas, como se detalla en la Tabla 1.

Tabla 1. Características geométricas de las secciones de carretera monitoreadas.

\begin{tabular}{|c|c|c|c|c|}
\hline Intersección estudiada & $\begin{array}{l}\text { Ancho de } \\
\text { carril }(\mathrm{m})\end{array}$ & $\begin{array}{c}\text { Ancho } \\
\text { vereda }(\mathrm{m})\end{array}$ & $\begin{array}{l}\text { Ancho de } \\
\text { parterre }(\mathrm{m})\end{array}$ & Intersección referencia \\
\hline $\begin{array}{l}\text { Av. Pumapungo desde Centrosur hasta } \\
\text { Lavadoras de Monay }\end{array}$ & 3 & 2 & 2 & $\begin{array}{l}\text { Av. Pumapungo y Max } \\
\text { Uhle }\end{array}$ \\
\hline $\begin{array}{l}\text { Av. Paraíso y Av. } 10 \text { de agosto desde el } \\
\text { Hospital Regional hasta el Parque Paraíso }\end{array}$ & 4 & 2 & 4 & Av. Paraíso y Arupo \\
\hline $\begin{array}{l}\text { Av. Paseo de los Cañaris entre Av. } \\
\text { Pumapungo y Viracochabamba }\end{array}$ & 3.5 & 2 & 2 & $\begin{array}{l}\text { Av. Paseo de los Cañaris y } \\
\text { Viracochabamba }\end{array}$ \\
\hline $\begin{array}{l}\text { Av. } 12 \text { de abril desde redondel José Peralta } \\
\text { hasta redondel Av. Paraíso }\end{array}$ & 3.45 & 2 & 0 & Paucarbamba y Los Fresnos \\
\hline $\begin{array}{l}\text { Av. } 10 \text { de agosto desde redondel Av. } 27 \text { de } \\
\text { febrero hasta redondel parque el Paraíso }\end{array}$ & 3.75 & 2 & 2 & Av. Paraíso y del Arupo \\
\hline $\begin{array}{l}\text { Av. } 12 \text { de abril desde Col. Sagrados } \\
\text { Corazones hasta calla Guayas }\end{array}$ & 3.75 & 2 & 3 & $\begin{array}{l}\text { Av. Loja y Remigio } \\
\text { Tamariz }\end{array}$ \\
\hline
\end{tabular}

\subsection{Determinación del flujo máximo obtenido en campo}

Los datos de flujo vehicular (q) de las avenidas analizadas se obtuvieron mediante la recopilación de datos en campo, utilizando el método Observador Moviéndose. Este método utiliza un vehículo de prueba, que circula entre el tráfico del tramo vial objeto de estudio, para determinar el tiempo empleado en recorrer el mismo. A partir de los datos recolectados se determinó la velocidad media (v), flujo vehicular (q) y las correspondientes densidades (k) observadas. Durante el monitoreo también se registra los vehículos pesados y livianos (Garber \& Hoel, 2014). Los datos tiempo de viaje, tiempo de parada, y 
vehículos que viajan, permitieron de calcular la velocidad (Ec. 2), flujo (Ec. 3) (Garber \& Hoel, 2014) y densidad (Ec. 4).

$$
\begin{gathered}
\mathrm{v}=\frac{\mathrm{d}}{\mathrm{t}} \\
\mathrm{q}=\frac{\text { Vehiculos }_{\text {c.o. }}+\text { Vehiculos }_{\mathrm{r} 1}-\text { Vehiculos }_{\mathrm{r} 2}}{\mathrm{~T}_{1}+\mathrm{T}_{2}} \\
\mathrm{k}=\frac{\mathrm{q}}{\mathrm{v}}
\end{gathered}
$$

dónde:

$\mathrm{d}=$ distancia del tramo recorrido en $\mathrm{km}$

$\mathrm{t}=$ tiempo empleado en horas

Vehículos $_{\text {c.o. }}=$ vehículos que circulan en el carril opuesto

Vehículos $\mathrm{s}_{\mathrm{r} 1}=$ vehículos que rebasan al vehículo observador

Vehículos $\mathrm{r}_{2}=$ vehículos que son rebasados por el vehículo observador

$\mathrm{T}_{1}=$ tiempo de viaje de ida

$\mathrm{T}_{2}=$ tiempo de viaje de regreso

$\mathrm{k}=$ densidad vehicular $(\mathrm{veh} / \mathrm{km} /$ carril $)$

$\mathrm{q}=$ flujo vehicular (veh/hora)

$\mathrm{v}=$ velocidad en kilómetros por hora $(\mathrm{kph}=\mathrm{km}$ por hora $)$

La determinación del flujo en cada sentido de circulación se realizó con datos del carril de desplazamiento del observador (vehículos que rebasan, vehículos rebasados), datos del carril opuesto (vehículos que viajan) y la suma de los tiempos de recorrido en ambos sentidos de circulación, para así obtener el valor de la densidad (k), expresada en vehículos por kilómetro. Posteriormente fueron aplicadas las relaciones fundamentales del flujo vehicular entre velocidad, flujo y densidad (Greenshields, 1935), para lo cual se relacionó linealmente la velocidad (v) y densidad (k) (Fig. 1), utilizando un conjunto de datos para diferentes condiciones de tránsito, estos datos fueron ajustados mediante mínimos cuadrados para obtener un modelo lineal, expresado en la Ec. (5).

dónde:

$$
\mathrm{v}_{\mathrm{e}}=\mathrm{v}-\left(\frac{\mathrm{v}_{\mathrm{l}}}{\mathrm{k}_{\mathrm{c}}}\right) \mathrm{k}
$$

$$
\begin{aligned}
& \mathrm{v}_{\mathrm{e}}=\text { velocidad media espacial }(\mathrm{kph}) \\
& \mathrm{v}_{\mathrm{l}}=\text { velocidad media espacial a flujo libre }(\mathrm{kph}) \\
& \mathrm{k}_{\mathrm{c}}=\text { densidad de congestionamiento }(\mathrm{veh} / \mathrm{km} / \mathrm{carril})(\text { Cal } \text { et al., } 1996)
\end{aligned}
$$

El modelo lineal de relación entre $\mathrm{v}$ y $\mathrm{k}$ permite identificar que cuando $\mathrm{v}$ es igual a cero, $\mathrm{k}$ tiene su valor máximo $\left(\mathrm{k}_{\mathrm{c}}\right)$ en la vía, es decir, los vehículos prácticamente se detienen, mientras que si $\mathrm{k}$ es cercana a cero (menor cantidad de vehículos) $\mathrm{v}$ es igual a la velocidad de flujo libre ( $\left.\mathrm{v}_{\mathrm{l}}\right)$. El VFL se define como la velocidad que se alcanzará en condiciones de bajo volumen y baja densidad de vehículos (TRB, 2000a). La capacidad de la vía, representada como el área del rectángulo de área máxima, corresponde al punto (E) (Fig. 1), el cual está ubicado exactamente en la mitad de la recta y su área representa el flujo máximo $\left(\mathrm{q}_{\mathrm{m}}\right)$, que representa el máximo flujo de vehículos que puede circular por las avenidas, conocido como capacidad de la vía (Greenshields, 1935). La ecuación (6) permitió obtener el flujo máximo para cada tramo de avenida estudiada.

$$
\mathrm{q}_{\mathrm{m}}=\frac{\mathrm{v}_{\mathrm{l}} \mathrm{k}_{\mathrm{c}}}{4}
$$




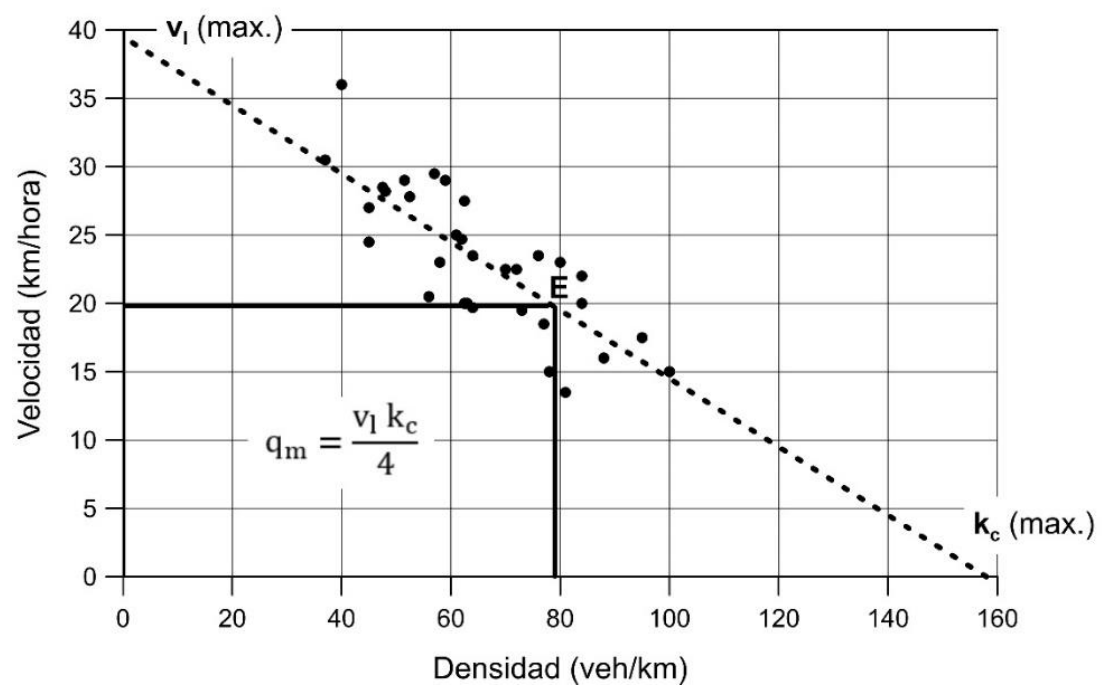

Figura 1. Relación lineal entre la densidad (k) y la velocidad (v) calculada en el tramo de vía de la Av. Paseo de los Cañaris entre Pumapungo y Viracochabamba (de igual manera se obtuvieron los valores y relaciones para las demás avenidas).

\subsection{Determinación del flujo máximo aplicando el Manual de Capacidad de Carreteras HCM}

HCM ha establecido seis niveles de servicio denominados LOS (Level Of Service) A, B, C, D, E, F que van del eficiente al inestable (Tabla 2).

Tabla 2. Los niveles de servicio para un segmento básico de autopista (HCM).

\begin{tabular}{cc}
\hline Nivel de servicio & $\begin{array}{c}\text { Rango de densidad } \\
(\mathrm{pc} / \mathrm{km} / \mathrm{carril})\end{array}$ \\
\hline $\mathrm{A}$ & $0-7$ \\
$\mathrm{~B}$ & $>7-11$ \\
$\mathrm{C}$ & $>11-16$ \\
$\mathrm{D}$ & $>16-22$ \\
$\mathrm{E}$ & $>22-28$ \\
$\mathrm{~F}$ & $>28$ \\
\hline
\end{tabular}

pc = vehículos pasajeros

Estos niveles de servicio se asignan de acuerdo con las condiciones preponderantes en la circulación vehicular (TRB, 2000a). El punto donde se cambia de nivel de servicio $\mathrm{E}$ a nivel de servicio $\mathrm{F}$ se conoce como Capacidad Vial y corresponde a un flujo de servicio $\left(\mathrm{v}_{\mathrm{p}}\right)$, expresado como $\mathrm{pc} / \mathrm{h} / \mathrm{carril}$ (vehículos pasajeros/hora/carril), calculado de manera experimental, cuyos resultados están en el HCM, en el cual se han estructurado gráficas de acuerdo con la tipología de las avenidas (Fig. 2) con valores de velocidad de automóviles y tasas de flujo de tráfico para encontrar el correspondiente nivel de servicio y capacidad $\left(\mathrm{q}_{\mathrm{m}}\right)$. En esta gráfica podemos observar, tabulados en el eje vertical, los valores correspondientes a la velocidad media de los vehículos, mientras que en el eje horizontal tenemos la tasa de flujo. Conociendo FFS (Free Flow Speed o Velocidad de Flujo Libre) se puede calcular la capacidad de la vía en el punto donde la línea, correspondiente a nuestra FFS, se encuentra con la línea que delimita el nivel de servicio E. La determinación de la velocidad de flujo libre FFS se obtiene mediante la ecuación (7) (TRB, 2000b).

$$
F F S=B F F S-f_{l w}-f_{l c}-f_{M}-f_{A}
$$

dónde:

- FFS: Free Flow Speed o Velocidad a Flujo Libre en kph. 
- BFFS: Base Free Flow Speed o Velocidad Base de Flujo Libre en kph. En este estudio se ha asumido una velocidad base de $50 \mathrm{kph}$, ya que es el límite de velocidad en las vías estudiadas.

- $\mathrm{f}_{\text {lw: }}$ ajuste para el ancho del carril (Tabla 23-4, TRB (2000b); a mayor ancho de carril menor penalidad).

- $\mathrm{f}_{\mathrm{lc}}$ : ajuste por distancia libre lateral total (Tabla 23-5, TRB (2000b); a mayor distancia libre menor será el valor de este ajuste).

- $f_{M}$ : ajuste por tipo de faja separadora central (Tabla 23-6, TRB (2000b); a mayor ancho de franja separadora menor penalidad en la velocidad).

- $\mathrm{f}_{\mathrm{A}}$ : ajuste por puntos de acceso (Tabla 23-7, TRB (2000b); a mayor cantidad de accesos por kilómetro, se incrementa el valor de la penalidad impuesta a la BFFS, en este caso se asumió una densidad de puntos de intersección a las avenidas locales de 12 intersecciones $/ \mathrm{km}$, lo que, referido a la Tabla 23-7, indica una penalidad de velocidad de $8 \mathrm{kph}$ ).

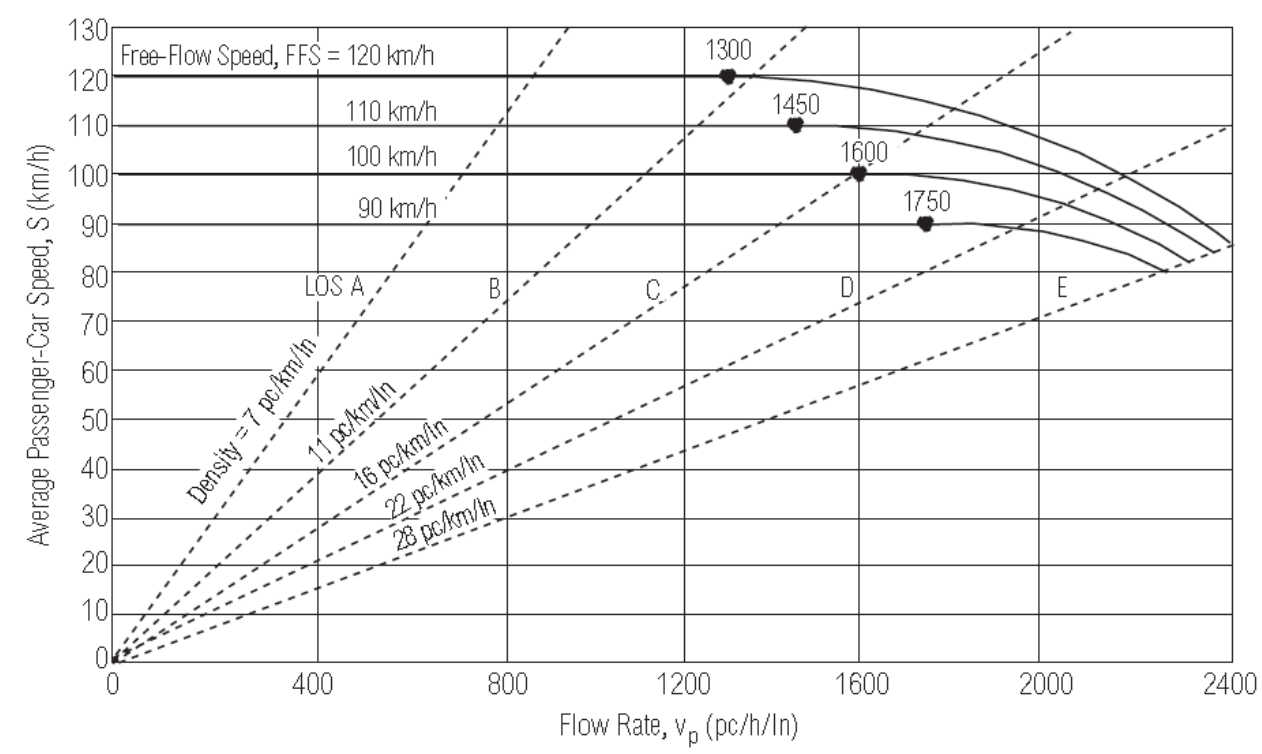

Figura 2. Cuadro de Niveles de Servicio y Capacidad (TRB, 2000b).

Nota: $\mathrm{v}_{\mathrm{p}}=$ tasa de flujo de servicio ( $\mathrm{pc} / \mathrm{h} / \mathrm{carril}$ ), y la capacidad varía según la velocidad de flujo libre. La densidad es de 2400, 2350, 2300 y $2250 \mathrm{pc} / \mathrm{km} /$ carril a velocidades de flujo libre de 120,110, 100 y $90 \mathrm{kph}$, respectivamente.

\section{RESULTADOS Y DISCUSIÓN}

\subsection{Comparación de las Velocidades de Flujo libre (FFS) determinadas en campo y mediante el modelo HCM}

Para el cálculo de la velocidad de flujo libre mediante el manual de HCM, se parte de una velocidad de flujo libre base de $50 \mathrm{kph}$ por efectos del límite permitido, se calculan lo factores inherentes a la geometría de las avenidas, se establecen los valores respectivos para FFS para luego contrastarlos con las velocidades obtenidas en el campo mediante la relación velocidad-densidad (Tabla 3).

Como se puede apreciar en la Tabla 3, hemos optado por realizar una operación estadística de promediar los valores obtenidos en cada una de las columnas de la tabla antes mencionada, sin embargo es necesario aclarar que la cantidad de muestras a promediar, con el fin de establecer un sesgo estadístico, es muy ínfimo, por tener solamente 6 casos ( 1 por cada avenida), además que existen valores, como el caso concreto de la Avenida 12 de abril (Av. José Peralta - Av. Paraíso), el cual claramente sobresale como un valor muy dispar debido a una singularidad ocurrida el día de la toma de los datos como es la obstrucción de vías aledañas. (Tabla 3; 4ta columna FFS/FFF ${ }_{\mathrm{HCM}}=1.49$ ). A pesar de que los valores de la velocidad de flujo son diferentes según los dos métodos aplicados, la relación entre los 
valores de $\mathrm{FFS}_{\text {campo }} \mathrm{FFS}_{\mathrm{HCM}}$ es muy cercana a 1.00 (promedio de los coeficientes = 1.06, DE 0.24). Lo observado permite concluir que los valores de FFS del método en campo y HCM son similares, estos resultados permiten en primera instancia validar el método HCM. Los resultados indican además que no se cumple lo dicho por HCM: “...por debajo de las condiciones básicas de una avenida multi-carril, FFS está 8 kph por encima de los límites de velocidad permitidos para velocidades permitidas entre 80 y $90 \mathrm{kph}$, y está $11 \mathrm{kph}$ por encima del límite permitido para límites de velocidad entre 65 y $70 \mathrm{kph}$..." (Tabla 23-5, TRB, 2000b). Se advierte una disparidad mayor de los resultados a medida que la velocidad base de flujo libre (BFFS) se aleja del parámetro básico de $100 \mathrm{kph}$, lo que no ocurre en nuestra localidad, según los datos obtenidos, existiendo una media de $6 \mathrm{kph}$ por debajo de la velocidad límite, contrario a lo propuesto por HCM. La fluctuación entre los valores obtenidos en las avenidas objeto de estudio, no son significativas puesto que todos oscilan entre 700 y $800 \mathrm{veh} / \mathrm{h}$, salvo dos excepciones que sobrepasan los 1,000 veh/h (Avenida 12 de abril) debidas principalmente al gran flujo que tiene esta avenida en sus días promedio; es decir por debajo de los 1,700 veh/hora

Tabla 3. Velocidades obtenidas mediante HCM versus obtenidas en campo y proporción.

\begin{tabular}{lcccc}
\hline \multicolumn{1}{c}{ Segmento vial estudiado } & $\begin{array}{c}\text { FFS }_{\mathrm{HCM}} \\
(\mathrm{kph})\end{array}$ & $\begin{array}{c}\mathrm{FFS}_{\text {campo }} \\
(\mathrm{kph})\end{array}$ & $\begin{array}{c}\text { Diferencia } \\
(\mathrm{kph})\end{array}$ & $\begin{array}{c}\text { FFS }_{\text {campo }} / \\
\text { FFS }_{\mathrm{HCM}}\end{array}$ \\
\hline Av. Pumapungo (Av. Max Uhle - Av. Rayoloma) & 31 & 26 & 5 & 0.83 \\
Av. 10 de agosto (Hosp. Regional - Parque Paraíso) & 42 & 44 & 2 & 1.04 \\
Av. Paseo de los Cañaris (Av. Pumapungo - & 41 & 35 & 6 & 0.86 \\
Viracochabamba) & & & \\
Av. 12 de abril (Av. José Peralta - Av. Paraiso) & 38 & 56 & 18 & 1.49 \\
Av.10 de Agosto (Av.27 de Febrero - Av.Paraiso) & 42 & 45 & 3 & 1.08 \\
Av. 12 de abril (Col. Sagrados Corazones - Calle & 42 & 44 & 2 & 1.05 \\
Guayas) & & & & \\
\hline Promedio & 39 & 42 & 6.00 & \multicolumn{2}{c}{1.06} \\
Desviación Estándar & 4.22 & 10.19 & 6.10 & 0.24 \\
\hline
\end{tabular}

\subsection{Comparación de las tasas de flujo máximo para el nivel de servicio (E) determinadas en campo y mediante el modelo HCM}

Con los resultados de las velocidades del inciso anterior y vamos a la Tabla 23-3 (TRB, 2000b), para el valor de velocidad de flujo libre (FFS), determinado mediante la fórmula que propone HCM, obtendremos una tasa de flujo máximo $\mathrm{q}_{\mathrm{m}}$ (automóviles/h/carril) (Tabla 4). Cabe indicar que el flujo obtenido en el campo es corregido por el factor de vehículos equivalente (FHV).

Debido a que las velocidades de campo y las obtenidas mediante el HCM fueron similares, se esperaría obtener también flujos máximos ( $\left.\mathrm{q}_{\mathrm{m}}\right)$ similares, sin embargo, esta situación no se da, $\mathrm{q}_{\mathrm{m} \cdot \mathrm{HCM}}$ se encuentra muy por encima de la tasa obtenida mediante los datos de campo (relación promedio $\mathrm{q}_{\mathrm{m} . c a m p o}$ / q $\mathrm{q}_{\mathrm{m} . \mathrm{HCM}}$ de 0.63 con una desviación estándar de 0.38). Dicho de otra manera, el flujo máximo obtenido mediante el HCM excede al valor obtenido con los datos de campo, ya que si invertimos la relación $\mathrm{q}_{\mathrm{m} . \mathrm{HCM}} /$ qm.campo, equivale a expresar $1 / 0.63=1.58$, indicándonos que la capacidad obtenida mediante HCM representa alrededor de un 58\% de exceso en su proyección para nuestra localidad. No se podría concluir y establecer este porcentaje como un valor fijo para ajustar este parámetro, puesto que, como indicamos anteriormente, el valor de $\left(\mathrm{q}_{\mathrm{m}}\right)$ obtenido en el campo, corresponde a condiciones de flujo particulares y ligadas a esos tramos específicamente, sin embargo, estos valores son suficientes para indicar que los valores obtenidos utilizando el método de HCM reflejan para este caso, una realidad vial distinta a la de la localidad. 
Tabla 4. Resultados de flujo $\left(\mathrm{q}_{\mathrm{m}}\right)$ máximo correspondiente a un nivel de servicio (E) obtenidos con HCM versus medidas y su proporción.

\begin{tabular}{|c|c|c|c|}
\hline Segmento vial estudiado & $\mathrm{qm} \cdot \mathrm{HCM}$ & $\begin{array}{l}\mathrm{q}_{\mathrm{m} \cdot \text { campo }} \\
\text { (corregido) }\end{array}$ & $\mathrm{q}_{\mathrm{m} . \text {.ampo }} / \mathrm{q}_{\mathrm{m} . \mathrm{HCM}}$ \\
\hline Av. Pumapungo (Av. Max Uhle - Av. Rayoloma) & 925 & 42 & 0.045 \\
\hline Av. Pumapungo (Av. Rayoloma - Av. Max Uhle) & 925 & 256 & 0.276 \\
\hline Av. 10 de agosto (Hosp. Regional - Parque Paraíso) & 1,225 & 801 & 0.654 \\
\hline Av. 10 de agosto (Parque Paraíso - Hosp. Regional) & 1,225 & 196 & 0.16 \\
\hline $\begin{array}{l}\text { Av. Paseo de los Cañaris (Av. Pumapungo - } \\
\text { Viracochabamba) }\end{array}$ & 1,200 & 758 & 0.631 \\
\hline $\begin{array}{l}\text { Av. Paseo de los Cañaris (Viracochabamba - Av. } \\
\text { Pumapungo) }\end{array}$ & 1,200 & 562 & 0.469 \\
\hline Av. 12 de abril (Av. José Peralta - Av. Paraíso) & 1,100 & 1,123 & 1.021 \\
\hline Av. 12 de abril (Av. Paraíso - Av. José Peralta) & 1,100 & 699 & 0.636 \\
\hline Av.10 de agosto (Av. 27 de febrero - Av. Paraíso) & 1,225 & 731 & 0.597 \\
\hline Av.10 de agosto (Av. Paraíso - Av.27 de febrero) & 1,225 & 781 & 0.638 \\
\hline Av. 12 de abril (Col. Sagrados Corazones - Calle Guayas) & 1,225 & 1,730 & 1.412 \\
\hline Av. 12 de abril (Calle Guayas - Col. Sagrados Corazones) & 1,225 & 1,218 & 0.994 \\
\hline Promedio & 1,150 & 741 & 0.63 \\
\hline Desviación estándar & 115 & 468 & 0.38 \\
\hline
\end{tabular}

\section{CONCLUSIONES}

Los resultados del presente trabajo reflejan valores inferiores de capacidad vial, en comparación con los resultados de la HCM (63\%). Similar situación a la encontrada en Autopistas de Argentina (Maldonado et al., 2012). Además, investigaciones sugieren que las diferencias se deben a que los conductores no están acostumbrados a circular a velocidades altas (Thenoux Zeballos, 2004). HCM advierte que sus ecuaciones deben ser comprobadas para vías donde la FFS es inferior a $60 \mathrm{kph}$ (Tabla 23-5, TRB, 2000b) como es el caso de estudio, sin embargo, anticipa que la FFS será mayor que el límite de velocidad. Opuesto a ello, los resultados obtenidos llevan a concluir que es menor en $6 \mathrm{kph}$.

Las velocidades de flujo libre calculadas el método HCM, son semejantes a las obtenidas en el campo con desviaciones en el orden de $6 \mathrm{kph}$, sin embargo, las capacidades de las vías son inferiores. Esto nos lleva necesariamente a la conclusión de que las curvas propuestas no se ajustan a la realidad local y deben ser ajustadas. La disparidad entre la tasa de flujo $\left(\mathrm{q}_{\mathrm{m}}\right)$, obtenida a través HCM, y su similar, obtenida de los datos de campo, nos abre el panorama hacia estudios futuros encaminados a encontrar maneras de apoyar nuestra investigación en HCM, sin dejar de lado el desarrollo de una propia metodología, Debería estudiarse con más profundidad los factores de tipo de conductor, el factor de vehículo equivalente y el factor de hora de máxima demanda. Probablemente es en estos factores donde se encuentre una explicación para la divergencia de capacidades (Thenoux Zeballos, 2004).

Recomendación: es necesario ampliar la cantidad de lecturas además de sistematizar la recolección de datos para de esta manera profundizar el estudio de los parámetros como: velocidad, flujo y densidad, y sus relaciones en la vialidad propia de nuestra urbe. En estudios futuros, se recomienda incluir más avenidas de similares características, con la finalidad de profundizar aún más el estudio y obtener resultados más concluyentes, y de ese modo establecer un modelo de ajuste aplicable en nuestra ciudad. Como propone Thenoux Zeballos (2004), un parámetro crítico en la capacidad de las carreteras son el conductor y su comportamiento, se considera que el comportamiento colectivo y su percepción de las ordenanzas y leyes es clave en el estudio del tránsito. HCM anticipa velocidades superiores a los límites de velocidad, sin embargo, debido a la ordenanza vigente en la ciudad los usuarios acatan estas restricciones, lo cual, a nuestro criterio, puede ser decisivo sobre la capacidad. 


\section{AGRADECIMIENTO}

A Juan Avilés, por su contribución con datos de campo recolectados y por su apoyo con documentos bibliográficos; al igual que a su esposa, Diana Morillo, por su ayuda en la edición del presente documento.

\section{BIBLIOGRAFÍA}

Arun, A., Velmurugan, S., Errampalli, M. (2013). Methodological framework towards roadway capacity estimation for Indian multi-lane highways. Procedia - Social and Behavioral Sciences, $104,477-486$.

Bessa Jr., J. E. (2009). Characterization of the traffic flow on two-lane rural highways in the state of São Paulo (in Portuguese). MSc thesis, Dissertação (mestrado), University of São Paulo, São Carlos School of Engineering, São Carlos, SP, Brazil. Disponible en http://www.teses.usp.br/ teses/disponiveis/18/18144/tde-04122009-150455/pt-br.php

Cal, R., Mayor, R., Cárdenas, J. (1996). Ingeniería de tránsito. (7ª ed.), 534 p. Ediciones Alfaomega, S. A. de C. V., México, D. F., México.

Elefteriadou, L., Lertworawanich, P. (2003). Defining, measuring and estimating freeway capacity. TRB 2003 Annual Meeting CD-ROM, 19 p. Retrieved from https://pdfs.semanticscholar.org/ ef5a/f84826310ddacbc111543b06d41e58a1fb50.pdf

Garber,N. J., Hoel, L. A. (2014). Traffic and highway engineering. (5 $5^{\mathrm{a}}$ ed.), 1296 p. Brooks/Cole, Pacific Grove, CA, USA.

Greenshields, B. D. (1935) A study of traffic capacity. Highway Research Board, 14, 448-477.

Hoogendoorn, S. P., Bovy, P. H. L. (2001). State-of-the-art of vehicular traffic flow modelling. Proc. of the Institution of Mechanical Engineers. Part I, Journal of Systems \& Control Engineering, 215(4), 283-303.

INEC. (2013). Estadísticas de transporte. INEC. Disponible en http://www.ecuadorencifras.gob.ec/ transporte/

Kondyli, A., George, B., Elefteriadou, L., Bonyani, G. (2017). Defining, measuring, and modeling capacity for the highway capacity manual. Journal of Transportation Engineering, Part A: Systems, 143(3), 1-12.

Lertworawanich, P., Elefteriadou, L. (2004). Evaluation of three freeway weaving capacity estimation methods and comparison to field data. Freeway Capacity, TRB 2004 Annual Meeting CD-ROM.

Luttinen, R. (2001). Percent time-spent-following as performance measure for two-lane highways. In: Transportation Research Records: Journal of the Transportation Research Board, No. 1776, TRB, National Research Council, Washington, D.C., pp. 52-59.

Maldonado, M. O., Herz, M., Galarraga, J. (2012). Modelacion de operación en carreteras argentinas y recomendaciones de ajustes al manual de capacidad HCM2010. Transportes, 20(3), 51-61.

Moreno, A. T., Llorca, C., Washburn, S. S., Bessa, J. E., Hale, D. K., Garcia, A. (2016). Modification of the Highway Capacity Manual two-lane highway analysis procedure for Spanish conditions. Journal of Advanced Transportation, 50(8), 1650-1665.

Romana, M., Pérez, I. (2006). Measures of effectiveness for level-of-service assessment of two-lane roads: An alternative proposal using a threshold speed. Transportation Research Record: Journal of the Transportation Research Board, 1988, 56-62.

Thenoux Zeballos, G. A. (2004). Aplicación y calibración del Highway Capacity Manual versión 2000 (HCM 2000) en una autopista chilena. Case study for the application of the Highway Capacity Manual (HCM 2000) the first Chilean highway. Revista Ingeniería de Construcción, 19(1), 2936.

TRB. (2000a). Highway capacity manual. Transportation Research Board, National Research Council, 
Washington, D. C., USA, 1200 p.

TRB. (2000b). Highway capacity manual 2000. Chapter 23 - Basic Freeway Segments 23-2:

Methodology. Transportation Research Board, Washington D.C., USA, Disponible en http://www.webpages.uidaho.edu/ce578/Course\%20materials/hcm2k23.pdf

Van As, C., Van Niekerk, A. (2004). The operational analysis of two-lane rural highways. Proc. Of the $23^{\text {rd }}$ Southern African Transport Conference (SATC 2004), pp. 622-633.

Van As, C. (2007). South African Highway Capacity Research. TRB Workshop Presentation. South African National Roads Agency, Limited. Disponible en http://www.nra.co.za/content/TRB6.pdf 